\title{
Probing the Molecular Orientation of a Single Conjugated Pol- ymer via Nano-gap SERS
}

\author{
Addison R. L. Marshall, ${ }^{a}$ Matthew Roberts ${ }^{\mathrm{b}}$, Johannes Gierschner, ${ }^{\mathrm{c}}$ Jean-Sebastien G. Bouil- \\ lard, ${ }^{* a, d}$ and Ali M. Adawi ${ }^{* a, d}$
}

a. Department of Physics and Mathematics, University of Hull, Cottingham road, HU6 7RX, UK.

b. Cambridge Display Technology Limited, Company number 02672530, Unit 12, Cardinal Park, Cardinal Way, Godmanchester, PE29 2XG, UK

c. Madrid Institute for Advanced Studies - IMDEA Nanoscience, Calle Faraday 9, Ciudad Universitaria de Cantoblanco, 28049 Madrid, Spain.

d. G. W. Gray Centre for Advanced Materials, University of Hull, Cottingham road, HU6 7RX, UK.

*E-mail: a.adawi@hull.ac.uk and j.bouillard@hull.ac.uk

KEYWORDS: Raman, SERS, Conjugated Polymer, DFT, Nano-gap.

\begin{abstract}
Determining the molecular orientation at the single molecule level is of key importance for a wide range of applications ranging from molecular electronic devices to biomedical applications. In this work Surface-Enhanced Raman Scattering (SERS) was used to probe the light emitting conjugated polymer F8-PFB at the single molecule level using nanoparticle on an extended metallic film nano-gap. The directional field enhancement of the nano-gap combined with Density Functional Theory (DFT) calculations was used to determine the orientation of the molecule. This analysis revealed that the spin-coated conjugated polymer preferentially aligns its molecular chains parallel to the metallic substrate. The integration of this approach in nanofabrication and synthesis will have a profound impact on different fields ranging from molecular electronic devices to biomedical applications.
\end{abstract}

\section{INTRODUCTION}

Conjugated polymers have been of significant interest due to their applications in optoelectronic devices such as organic light emitting diodes, photovoltaics, lasers, as well as bio-probes and sensors. ${ }^{1-3}$ The optical and electronic properties of conjugated polymer thin-films are highly dependent on the processing and deposition conditions, ${ }^{4,5}$ illustrating the strong relationship between morphology and photophysics. This relationship can be revealed by studying these materials at the single molecule level which is commonly done using single molecule fluorescence spectroscopy. ${ }^{6}$ Although this technique provides a wealth of information regarding the processes that contribute to excited state relaxation and energy transfer between chromophores of large molecules, it brings limited structural/conformational information about these materials. Raman spectroscopy, on the other hand, has been shown to be a

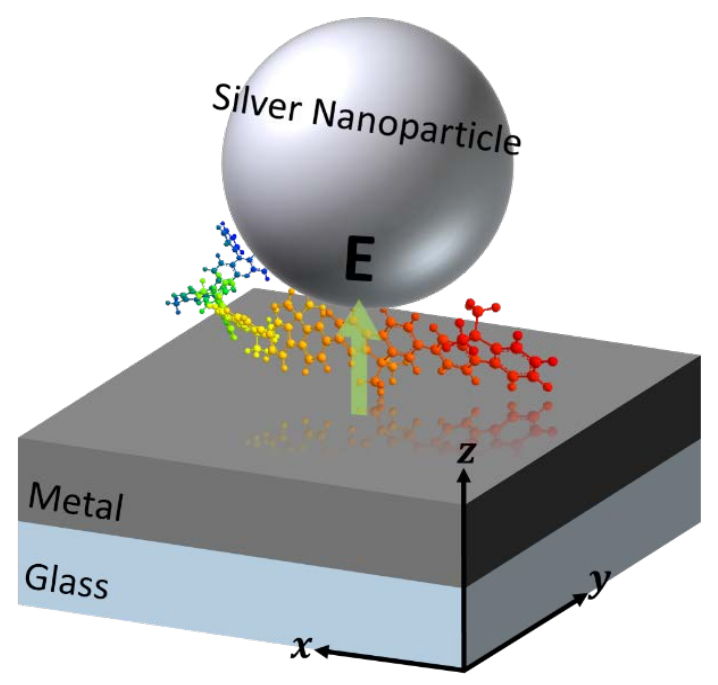

Figure 1. Schematic representation of the SERS plasmonic nano-gap investigated in this work.

powerful technique for materials characterization, providing detailed structural information in a label free and noninvasive manner.7,8

The use of Raman scattering for molecular identification down to the single molecule level can be challenging and requires large enhancements of the electromagnetic (EM) fields in the analyte local environment. By coupling Raman spectroscopy with plasmonic devices, SERS (Surface Enhanced Raman Scattering) has been shown to provide molecular fingerprints unique to the material under investigation down to the single molecule regime. ${ }^{9-13}$

On the other hand, determining the molecular orientation of an analyte in situ is of key importance in a range of applications. In optoelectronics, molecular orientation is important for the development of high performance devices, in particular of organic light-emitting diodes (OLEDs), organic field-effect transistors (OFETs) and molecular 
switches. 4,14,15 Furthermore, in biomedical sciences, knowing the molecular orientation enables conformation changes in DNA to be monitored, ${ }^{16}$ as well as probing in more details the forward rates of cell-bound receptor-ligand binding which has been shown to be strongly influenced by molecular orientation. ${ }^{17,18}$ Additionally, by understanding the interaction mechanisms of biomolecules the possibility for drug development also increases significantly. ${ }^{19}$

Polyfluorene (PF) based materials generated special attention, and the importance of the chain orientations with respect to the substrate were highlighted by various studies. ${ }^{20,21}$ In bulk films this orientation can be recovered by specular or grazing incidence $\mathrm{x}$-ray diffraction for polycrystalline samples, ${ }^{22}$ or by polarized techniques (optical absorption, photoluminescence, Raman or x-ray microscopy). ${ }^{21,23}$

There is however an increasing need to probe molecular orientations in materials at a single molecule level. This can be done by azimuthally or radially polarized laser beams, ${ }^{24,25}$ or by SERS. The orientation of small molecular targets through SERS has already been recovered in previous studies. ${ }^{19,26-32}$ However, using SERS to determine the molecular orientation of large molecules such as conjugated polymers is still a real challenge as for large molecules the molecular sizes are typically larger than the enhancement volume. Additionally molecular diffusion leads to changes in molecular conformations and orientations within the enhancement volume resulting in selective Raman enhancement over time. These two effects combine to make determining the orientation of large molecules more challenging. ${ }^{33,34}$

Up to now only two studies report on SERS spectra of single conjugated polymer chains, ${ }^{35,36}$ where simple nanostructured silver films were used to provide the necessary electromagnetic enhancement. However, the random nature of the enhanced electromagnetic field associated with these films averages out the inherited information on molecular orientation contained within the SERS signal. The nano-gap architecture used throughout this study (see Figure 1 and Figure S1)) was shown to preferentially enhance Raman modes which are aligned with the nano-gap field. ${ }^{32}$ This preferential alignment in the enhanced Raman modes allows to determine the orientation of the molecule relative to the nano-gap field.

In this work, the orientation of a single chain of the PFbased conjugated co-polymer, poly (9,9'-dioctylfluorene-cobis-N,N'-(4-butylphenyl)-bis-N,N'-phenyl-1,4-phenylenediamine), otherwise known as F8-PFB (0.95:0.05 F8:PFB and see Figure 2(a)) was recovered using the well-defined electromagnetic field enhancement direction in a plasmonic nano-gap formed between a silver nanoparticles and a silver film (see Figure 1). Silver nanoparticles of $118 \mathrm{~nm}$ in diameter were coupled to a $100 \mathrm{~nm}$ thick silver film to create the plasmonic nano-gaps which have been shown to be optimized for SERS excitation at a wavelength of 532nm. ${ }^{32}$ By combining the single molecule SERS spectra from those nano-gaps with Density Functional Theory (DFT) calculations, the molecular orientation of a single chain of F8:PFB was determined. The results of this analysis clearly demonstrate that spin-coated conjugated polymers tend to align their molecular chains parallel to the metallic substrate.

\section{METHODS}

The plasmonic nano-gaps were fabricated on glass slides which were first sequentially cleaned in solutions of acetone, isopropanol and deionized water for 10 minute periods in an ultrasonic bath. After cleaning, the slides were thermally coated with a silver film of average thickness 100 $\mathrm{nm}$, forming the bottom of the nano-gap (Figure 1). The conjugated polymer F8-PFB $\left(\mathrm{M}_{\mathrm{w}}=355 \mathrm{kDa}\right.$ and 0.95:0.05 F8:PFB), dissolved in toluene at concentrations ranging between $2.8 \times 10^{-5} \mathrm{M}$ to $2.8 \times 10^{-10} \mathrm{M}$ (ref. 6,13,35), was deposited via spin coating. Lastly, silver nanoparticles of average diameter $118 \mathrm{~nm}$ (Sigma-Aldrich) were spin-coated onto the surface of the polymer coated film from a solution of ethanol at a concentration of $2.7 \times 10^{6}$ particle. $L^{-1}$. This concentration was used to obtain several microns spacing between the nanoparticles (see Figure S2), therefore ensuring that the nano-gaps are isolated from one another.

SERS measurements were performed using a home built Raman setup with a $532 \mathrm{~nm}$ laser line using off-resonance excitation (see Figure 2(b)). The measurements were acquired via direct excitation through a $50 \times$ magnification objective lens with Numerical Aperture NA $=0.55$ (Mitutoyo M Plan Apo 50X). The collected Raman signal was then focused onto the entrance slit of an iHR320 Horiba spectrometer where it was dispersed using a 1200 line. $\mathrm{mm}^{-1}$ grating onto a liquid nitrogen cooled Symphony CCD. Rayleigh scattering was filtered from the Stokes scattered light using two Kaiser holographic notch and super-notch filters centered at a wavelength of $532 \mathrm{~nm}$.

The experimental Raman scattering spectra were complemented with DFT calculations, done on F8 ${ }_{n}$ oligomers $(\mathrm{n}=$ $1,2,3,6)$, PFB and the F8 3 PFB co-oligomer. The geometries of $\mathrm{F}_{\mathrm{n}}$ were optimized in the $\mathrm{C}_{2}$ point group. For all calculations, the B3LYP functional and $6-311 \mathrm{G}^{*}$ basis set were employed as defined in the Gaussian 09 program package. ${ }^{37}$ As the PFB unit contributes only very little to the Raman spectrum (vide infra), and in experiment the PFB content is only $5 \%$, interpretation of the Raman spectra were based on the F8 $n$ oligomers. Raman frequencies $v_{i}$ at the F8 $\infty$ polymer limit were obtained by an exponential extrapolation of $v_{i}$ vs. $\mathrm{N}^{-1}$, i.e. the inverse number of double bonds along the shortest path between the terminal C-atoms. ${ }^{38}$ As the frequencies of the relevant modes at the polymer limit are practically identical with those of F8 (vide infra), the analysis of the Raman tensors relied on F86. For this, the absolute values of the atomic displacements (see Figure S2) were summed, weighted by the square root of the mass; using this information, the inclination against the axis defined relative to the terminal carbon atoms in the chain was resolved.

\section{RESULTS AND DISCUSSION}

Figure 2(c) shows the Raman signal from F8-PFB at a concentration of $2.8 \times 10^{-5} \mathrm{M}$ on a glass substrate which provides a full fingerprint spectrum of the material. The most prominent Raman mode of the material is observed at $1605 \mathrm{~cm}^{-1}$ which, using DFT calculations on $\mathrm{F}_{6}$, can be attributed to a mode corresponding to combined phenyl inter- and intra- 
ring $\mathrm{C}-\mathrm{C}$ stretches (vide infra). Additional, albeit less intense Raman modes can be identified in the range between 1100 $1500 \mathrm{~cm}^{-1}$ (with prominent bands at 1136, 1258, 1281, $1350,1421 \mathrm{~cm}^{-1}$ ). The DFT analysis assigns these bands to normal modes combining intra- and inter-ring $\mathrm{C}$-C stretching, and $\mathrm{C}-\mathrm{H}$ and $\mathrm{C}-\mathrm{C}$ bending motions.
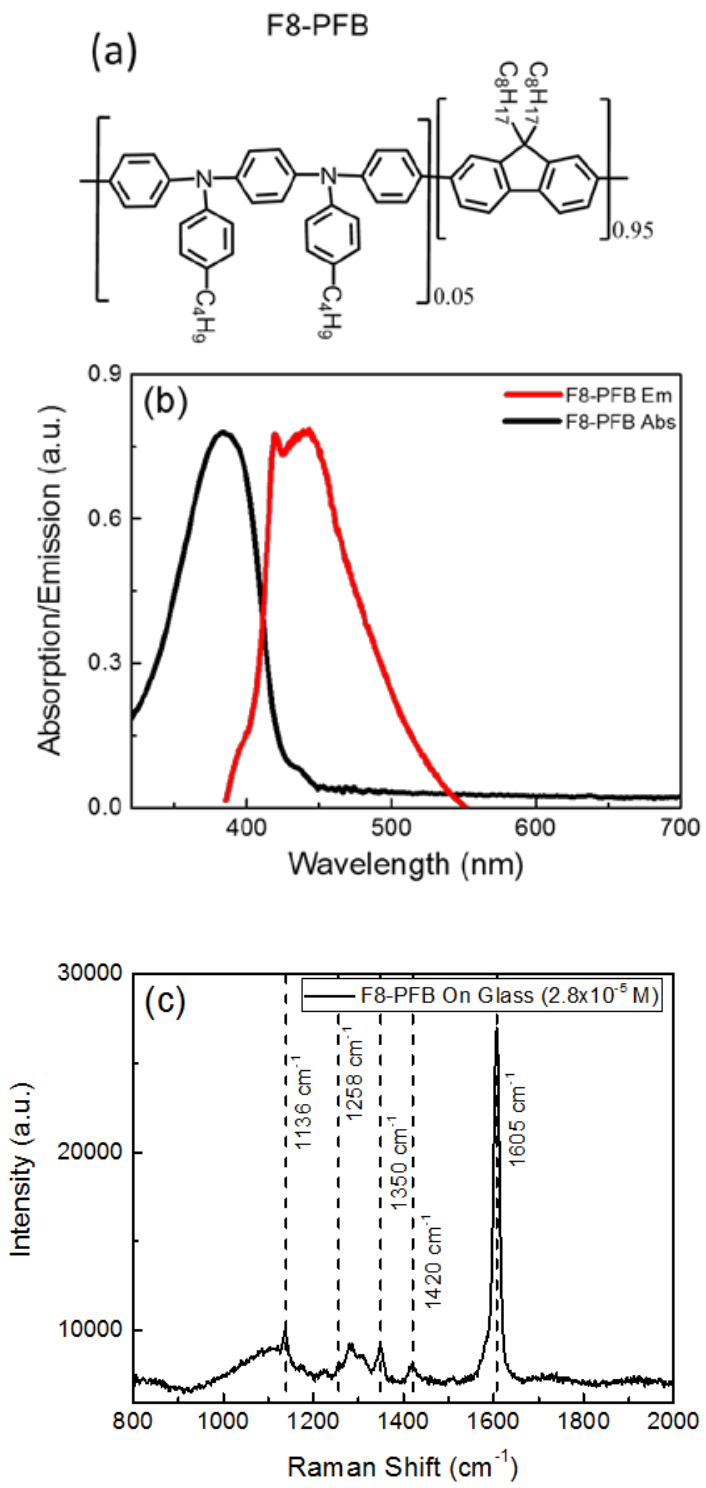

Figure 2. (a) The chemical structure of F8-PFB. (b) F8-PFB Absorption (black line) and Emission (red dotted line) spectra peaking at $383 \mathrm{~nm}$ and $463 \mathrm{~nm}$ respectively. (c) F8-PFB Raman spectrum taken from a thin film on glass at $2.8 \times 10^{-5} \mathrm{M}$.

The SERS spectrum of F8-PFB was then studied inside the nano-gaps at different concentrations. As the concentration of F8-PFB is systematically lowered with each sample, a corresponding decrease in intensity of the SERS peaks is observed and attributed to the reducing number of molecules contributing to the Raman signal (Figure 3(a)). The decrease in concentration also reduces the film thickness (Figure 3(a)) which we measured at each concentration using surface profilometry. However, for concentrations lower than $3 \times 10^{-9} \mathrm{M}$, the intensity of the Raman signal no longer decreases with concentration but remains constant (Figure 3(b)), suggesting that, for such low concentrations, the Raman signal is attributed to a single (macro)molecule in the nano-gap.
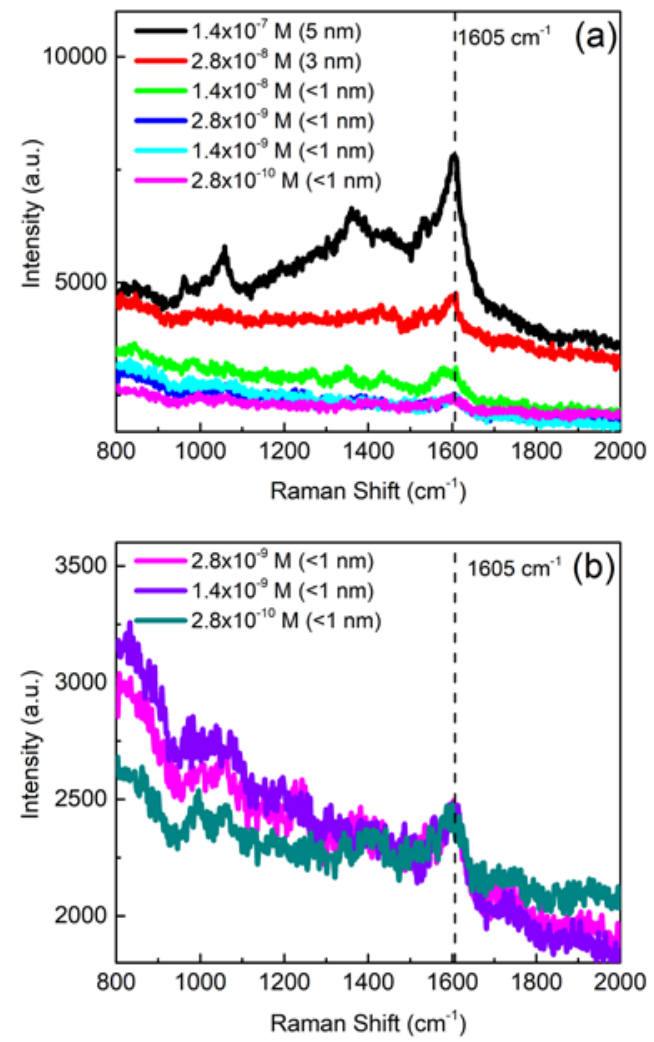

Figure 3. SERS concentration study of F8-PFB for 118 $\mathrm{nm}$ silver nanoparticles on a silver film (estimated thickness in parentheses) taken at $532 \mathrm{~nm}$ excitation with 3 s collection time. (a) The full concentration range and (b), the point at which intensity no longer drops.

To further confirm the single molecule regime, 10 consecutive SERS measurements were taken from nano-gaps with two different polymer concentrations, $2.8 \times 10^{-8} \mathrm{M}$ and $2.8 \times 10^{-9} \mathrm{M}$, over a period of 30 seconds. As can be seen in Figure 4, for the higher concentration of $2.8 \times 10^{-8} \mathrm{M}$, the intensity of the $1605 \mathrm{~cm}^{-1} \mathrm{C}-\mathrm{C}$ stretching mode is constant. However, lowering the concentration one order of magnitude to $2.8 \times 10^{-9} \mathrm{M}$, leads to the Raman signal to blink in intensity over time. In the configuration considered here, with the molecule in direct contact with metallic surfaces, this characteristic behavior of single molecule events can be explained by photoionization via charge-transfer states. ${ }^{39-41}$ 

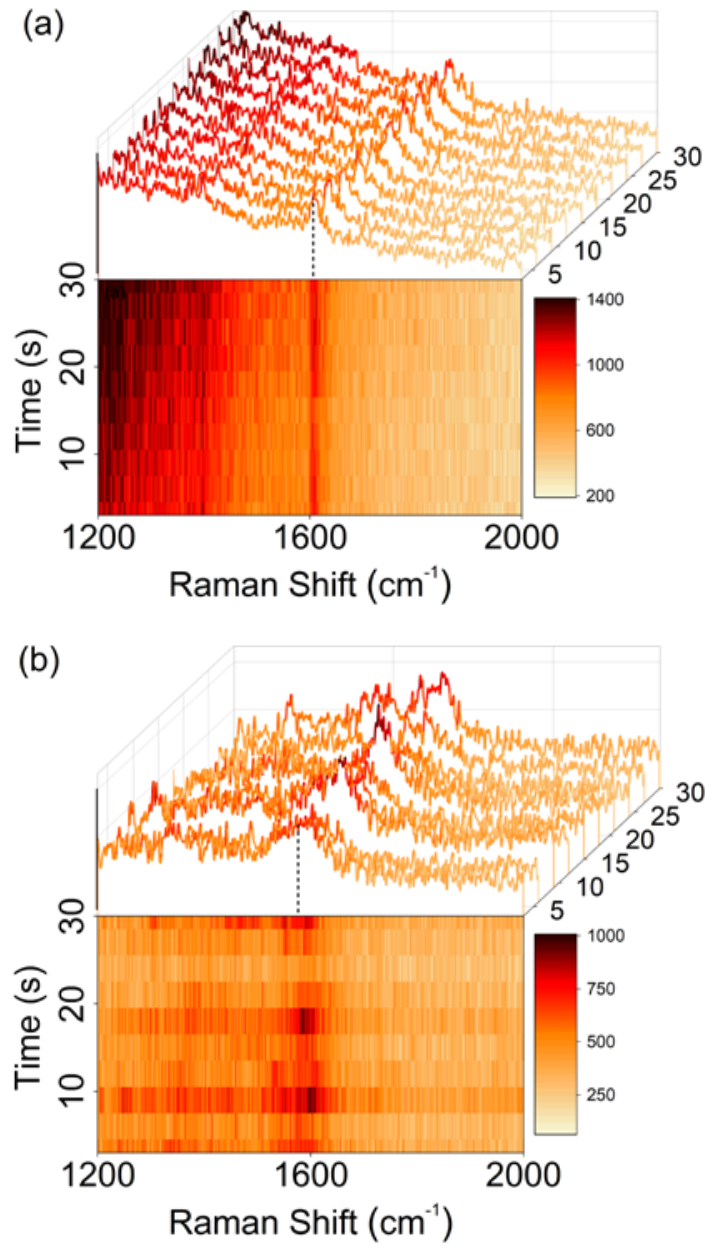

Figure 4. 10 consecutive SERS measurements for F8PFB at (a) $2.8 \times 10^{-8} \mathrm{M}$ and (b) $2.8 \times 10^{-9} \mathrm{M}$. The C-C stretching mode fluctuates in intensity with each successive measurement.

To explore the single molecule Raman spectra of F8-PFB further, typical single molecule SERS spectra of F8-PFB measured from nano-gaps at concentration $2.8 \times 10^{-9} \mathrm{M}$ are shown in Figure 5 (panels 2-6), along with the corresponding Raman spectrum at high concentration for comparison (panel 1). In contrast to the high concentration Raman spectrum, which shows one strong peak at $1605 \mathrm{~cm}^{-1}$, the single molecule data clearly shows two Raman peaks, centered at $1605 \mathrm{~cm}^{-1}$ and $1556 \mathrm{~cm}^{-1}$ respectively.

The DFT calculations demonstrate that the two peaks observed experimentally are dominated each by single vibrations (see Figure 6(a)); furthermore, PFB vibrations are of low intensity (see inset of Figure 6(a)) and can be thus safely neglected. The $\mathrm{R}_{1}$ frequency remains fairly constant with $\mathrm{n}$, giving $1617 \mathrm{~cm}^{-1}$ at the polymer limit (see Figure 6(b)); it combines intra-fluorene stretching and asymmetric breathing motions (Figure 7 (a)) and is polarized at a $46^{\circ}$ angle to the terminal C-atoms of the polyfluorene chain (see Figure $7(b)$ ). $R_{2}$ decreases with $n$, but saturates fast towards the polymer limit, being essentially the same as for $n$ $=6\left(1642 \mathrm{~cm}^{-1}\right) \cdot \mathrm{R}_{2}$ combines fluorene inter- and intra-ring $\mathrm{C}-\mathrm{C}$ stretches (Figure $7(\mathrm{a})$ ) and is polarized at a $17^{\circ}$ angle to the terminal $\mathrm{C}$-atoms of the polyfluorene chain (Figure 7(b)); the enclosed angle between $\mathrm{R}_{1}$ and $\mathrm{R}_{2}$ is $50^{\circ}$ (Figure $7(\mathrm{c})$ ).

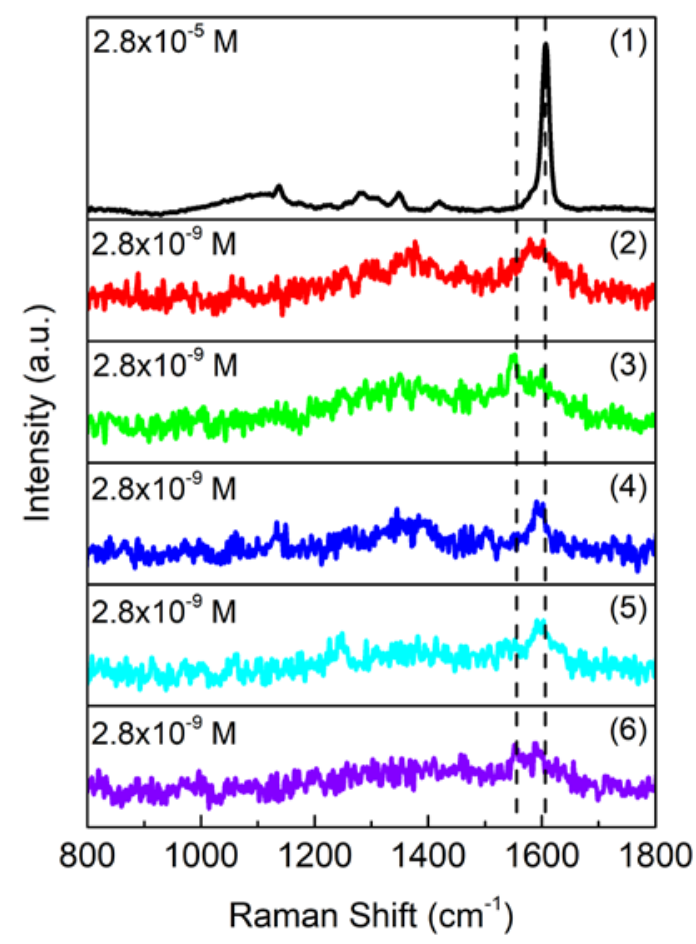

Figure 5. Raman spectra from F8-PFB at $2.8 \times 10^{-5} \mathrm{M}$ on glass (panel 1), and SERS spectra from F8-PFB at $2.8 \times 10^{-9} \mathrm{M}$ within the nano-gaps between a silver nanoparticle and silver film (panels 2-6).

As mentioned earlier, in SERS, the relative intensities of the peaks depend on the molecular orientation relative to the nano-gap field. For each analyte molecule, the SERS signal intensity, $I_{S E R S}$ is proportional to $\cos ^{2}(\theta), 42$ and can be written as follows:

$$
I_{S E R S}=I_{\text {Raman }} \cdot G \cdot \cos ^{2} \theta
$$

Where $\theta$ is the angle between the driving electric field and the direction of Raman tensor of the mode (Figure 7b), $I_{\text {Raman }}$ is the intensity of the Raman mode in the absence of plasmonic structure, and $G$ is the SERS enhancement factor. $^{32}$

Using equation (1), the SERS enhancements in $\mathrm{R}_{1}$ $I_{S E R S_{R 1}}$ and $\mathrm{R}_{2} I_{S E R S_{R 2}}$ can be written as:

$$
\begin{gathered}
I_{S E R S_{R 1}}=I_{D F T_{R 1}} \cdot G \cdot \cos ^{2}(\theta)(2) \\
I_{S E R S_{R 2}}=I_{D F T_{R 2}} \cdot G \cdot \cos ^{2}(\theta+50)(3)
\end{gathered}
$$

The ratio between $I_{S E R S_{R 1}}$ and $I_{S E R S_{R 2}}$ can therefore be expressed as:

$$
\frac{I_{S E R S_{R 1}}}{I_{S E R S_{R 2}}}=\frac{I_{D F T_{R 1}}}{I_{D F T_{R 2}}} \cdot \frac{\cos ^{2}(\theta)}{\cos ^{2}(\theta+50)}
$$


(a)

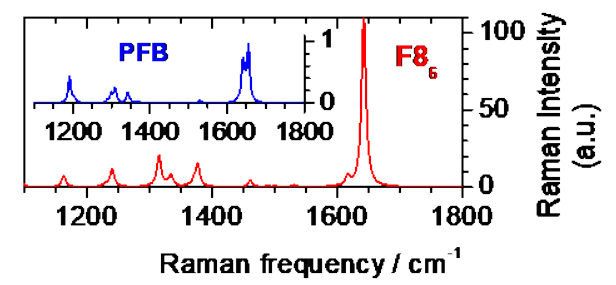

(b)

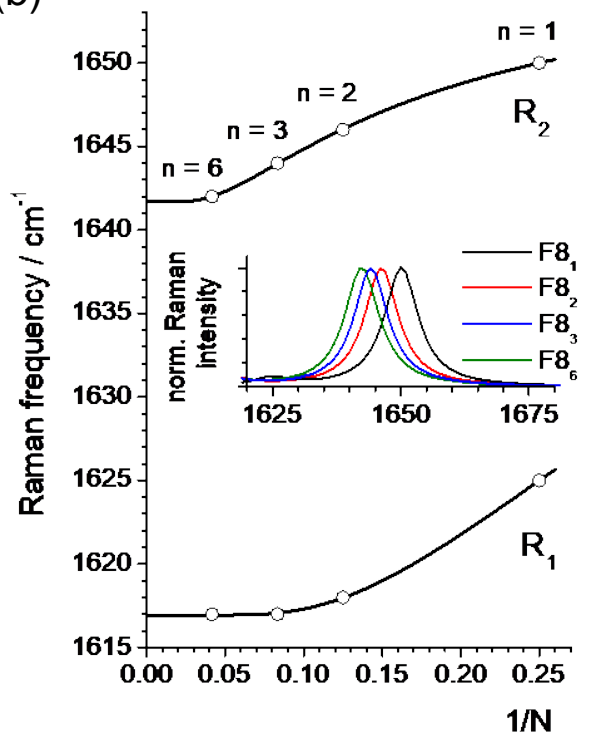

Figure 6. DFT calculations demonstrating (a) Raman spectrum of F8 6 (PFB as inset). (b) Peak shift of $\mathrm{R}_{1}$ and $\mathrm{R}_{2}$ Raman frequencies towards the polymer limit $\left(\mathrm{N}^{-1}=\right.$ 0 ; see Method section).

By combining equation (4) with the single molecule spectra of figure 5 , it is possible to determine the angle $\theta$, and therefore the orientation of the single polymer chain within each nano-gap (table 1).

Table 1. Calculating the orientation of the target analyte with respect to the cavity using experimental SERS intensity for peaks $R_{1}$ and $R_{2}$ at single molecule spectra from Figure 5 .

\begin{tabular}{|l|l|l|l|}
\hline Intensity $\mathrm{R}_{1}$ & Intensity $\mathrm{R}_{2}$ & $\theta\left(\mathrm{R}_{1}\right)$ & $\theta\left(\mathrm{R}_{2}\right)$ \\
\hline 328 & 337 & $28^{\circ}$ & $78^{\circ}$ \\
\hline 589 & 315 & $35^{\circ}$ & $85^{\circ}$ \\
\hline 207 & 305 & $23^{\circ}$ & $73^{\circ}$ \\
\hline 256 & 330 & $25^{\circ}$ & $75^{\circ}$ \\
\hline 342 & 234 & $32^{\circ}$ & $82^{\circ}$ \\
\hline
\end{tabular}

Table 1 shows that the angle for the $\mathrm{R}_{2}$ band calculated from the DFT data and SERS spectra is between $73^{\circ}$ to $85^{\circ}$ relative to nano-gap field (z-axis in figure $7 \mathrm{c}$ ). On average
$R_{2}$ makes an angle of $11^{\circ}$ relative to the substrate. Therefore, when considering the main axis of the $\mathrm{R}_{2}$ tensor in figure $7 \mathrm{~b}$ as being $17^{\circ}$ with respect to the long polymer axis, our calculations indicate that the spin-coated F8-PFB molecules align their molecular chain almost parallel to the substrate within the nano-gap. This is further supported by the comparable intensities of $\mathrm{R}_{1}$ and $\mathrm{R}_{2}$ in SERS which arise from their respective projection of their dipole derivative vectors along the nano-gap field.

(a)
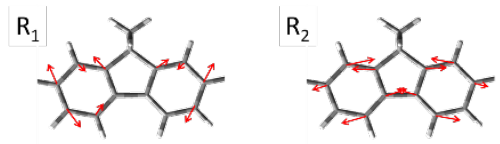

(b)
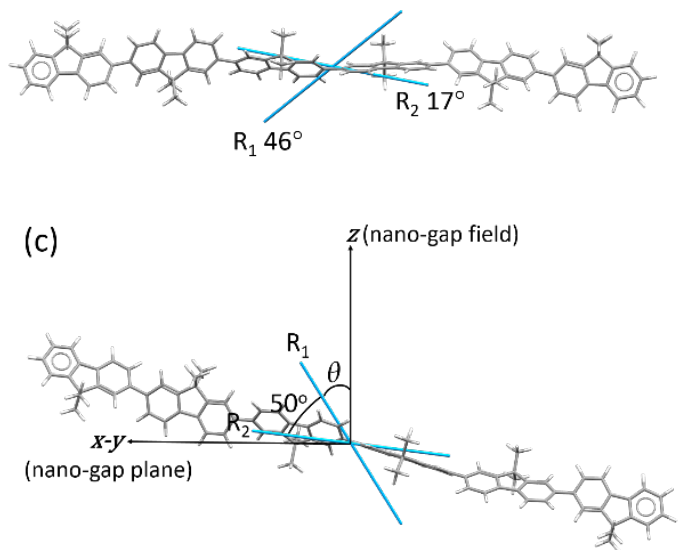

Figure 7. (a) Relative atomic displacements of the Catoms in the central fluorene units for $\mathrm{R}_{1}$ and $\mathrm{R}_{2}$. (b) Main axis of the polarizability tensor of $R_{1}$ and $R_{2}$ for F86: (angles vs. terminal C-C). (c) Orientation of the target analyte with respect to the nano-gap field.

It should be stressed that the high in-plane orientation found in the current work is much larger than in ellipsometry studies e.g. on spin-coated poly-dicoctylfluorene films. ${ }^{21}$ We attribute this mainly to the much thicker films in these experiments; furthermore the substrate might have a significant effect. The observed differences high-light the advantage of the SERS-based method; i.e. to obtain local information on polymer organization at a single molecular level.

\section{CONCLUSION}

In conclusion, we have shown that through combining SM-SERS spectroscopy and DFT calculations, it is possible to determine the molecular orientation of conjugated polymers from plasmonic nano-gaps at concentrations demonstrating single molecule level characteristics. Our analysis revealed that spin-coated conjugated polymers preferentially align their molecular chains almost parallel to the substrate. The work shown in this study provides a better understanding of the interfacial interaction of conjugated polymers with metallic surfaces and offers a powerful tool to optimizing conjugated polymers based devices such as OLEDs, OFETs and molecular switches. In parallel, in situ retrieval of the molecular orientation and confirmation will have profound impacts on biomedical sciences, allowing for, 
amongst others, DNA monitoring, probing of forward rates of cell-bound receptor-ligand binding, and enhanced drug development and delivery.

\section{ASSOCIATED CONTENT}

Supporting Information.

The Supporting Information is available free of charge via the Internet at http://pubs.acs.org."

Calculated field enhancement, typical dark-field imaging of the sample, calculated Raman modes for F8-PFB (pdf)

\section{AUTHOR INFORMATION}

\section{Corresponding Authors}

*E-mail address:j.bouillard@hull.ac.uk

*E-mail address: a.adawi@hull.ac.uk

\section{ORCID}

Johannes Gierschner: oooo-ooo1-8177-7919

Jean-Sebastien G. Bouillard: oooo-0oo2-6942-1749

Ali M. Adawi: oooo-0oo1-6850-5679

\section{Author Contributions}

The manuscript was written through contributions of all authors.

\section{Funding Sources}

Any funds used to support the research of the manuscript should be placed here (per journal style).

- University of Hull for A. Marshall's PhD scholarship

- UK EPSRC Grant. No. EP/Lo25078/1 "Self assembly of two dimensional colloidal alloys for metamaterials applications"

- $\quad$ European COST Action Nano-spectroscopy, MP13o2

- Campus of International Excellence (CEI) $\mathrm{UAM}+\mathrm{CSIC}$

- MINECO-FEDER project CTQ2017-87054 "Severo Ochoa" program for Centers of Excellence in R\&D (MINECO, grant SEV2016-o686)

\section{Notes}

The Authors declare no competing financial interest.

\section{ACKNOWLEDGMENT}

We thank the University of Hull for the PhD scholarship which funded this project. This work was partially supported by the UK EPSRC through Grant. No. EP/L025078/1 "Self assembly of two dimensional colloidal alloys for metamaterials applications" and the European COST Action Nano-spectroscopy, MP1302. Additionally, we would like to thank Cambridge Display Technology (CDT) Limited for providing us with the F8-PFB polymer. J.G. thanks B. Milían-Medina, Valencia for technical assistance with the Raman calculations. The work at IMDEA was supported by the Spanish Ministerio de Economiá y Competitividad (MINECO-FEDER project CTQ2017-87054), by the "Severo Ochoa" program for Centers of Excellence in R\&D (MINECO, grant SEV2016-0686) and by the Campus of International Excellence (CEI) UAM+CSIC.

\section{ABBREVIATIONS}

SM; Single Molecule; SERS, Surface Enhanced Raman Spectroscopy; DFT, Density functional Theory.

\section{REFERENCES}

(1)

(10)

(10

Ostroverkhova, O. Handbook of Organic Materials for Optical and (Opto)Electronic Devices, 1st ed.; Woodhead Publishing, 2013.

Hu, W.; Bai, F.; Gong, X.; Zhan, X.; Fu, H.; Bjornholm, T. Organic Optoelectronics, 1st ed.; Wiley-VCH: Germany, 2013. Materials, R. S. C. S. Supramolecular Materials for OptoElectronics, 1st ed.; Koch, N., Ed.; Smart Materials Series; Royal Society of Chemistry: Cambridge, 2014.

Dykstra, T. E.; Hennebicq, E.; Beljonne, D.; Gierschner, J.; Claudio, G.; Bittner, E. R.; Knoester, J.; Scholes, G. D. Conformational Disorder and Ultrafast Exciton Relaxation in PPV-Family Conjugated Polymers. J. Phys. Chem. B 20o9, 113. Olivier, Y.; Niedzialek, D.; Lemaur, V.; Pisula, W.; Müllen, K.; Koldemir, U.; Reynolds, J. R.; Lazzaroni, R.; Cornil, J.; Beljonne, D. 25th Anniversary Article: High-Mobility Hole and Electron Transport Conjugated Polymers: How Structure Defines Function. Adv. Mater. 2014, 26, 2119-2136. Khalil, G. E.; Adawi, A. M.; Fox, A. M.; Iraqi, A.; Lidzey, D. G. Single Molecule Spectroscopy of Red- and Green-Emitting Fluorene-Based Copolymers. J. Chem. Phys. 2009, 130, 2-9.

Maier, S. A. Plasmonics: Fundamentals and Applications; Springer: New-York, 2007.

Heinz Raether. Surface Plasmons on Smooth and Rough Surfaces and on Gratings, 1st ed.; Springer Tracts in Modern Physics; Springer Berlin Heidelberg: Berlin, 1988; Vol. 111.

Le Ru, E. C.; Etchegoin, P. G. Principles of Surface Enhanced Raman Spectroscopy and Related Plasmonic Effects, 1st ed.; Elsevier Ltd: Amsterdam, 2009.

Lee, H. M.; Jin, S. M.; Kim, H. M.; Suh, Y. D. Single-Molecule Surface-Enhanced Raman Spectroscopy: A Perspective on the Current Status. Phys. Chem. Chem. Phys. 2013, 15, 52765287.

Zhang, R.; Zhang, Y.; Dong, Z. C.; Jiang, S.; Zhang, C.; Chen, L. G.; Zhang, L.; Liao, Y.; Aizpurua, J.; Luo, Y.; Yang, J. L.; Hou J. G. Chemical Mapping of a Single Molecule by PlasmonEnhanced Raman Scattering. Nature 2013, 498, 82-86.

Etchegoin, P. G.; Le Ru, E. C.; Fainstein, A. Bi-Analyte Single Molecule SERS Technique with Simultaneous Spatial Resolution. Phys. Chem. Chem. Phys. 2011, 13, 4500.

Kneipp, K.; Wang, Y.; Kneipp, H.; Perelman, L. T.; Itzkan, I.; Dasari, R. R.; Feld, M. S. Single Molecule Detection Using Surface-Enhanced Raman Scattering (SERS). Phys. Rev. Lett. 1997, 78, 1667-1670.

Song, H.; Reed, M. A.; Lee, T. Single Molecule Electronic Devices. Adv. Mater. 2011, 23, 1583-1608.

Jurow, M.; Schuckman, A. E.; Batteas, J. D.; Drain, C. M. Porphyrins as Molecular Electronic Components of Functional Devices. Coord. Chem. Rev. 2010, 254, 2297-2310. Spuhler, P.; Zhang, X.; Monroe, M.; Greenspun, J.; Unlu, M. S.; Sola, L.; Chiari, M. Precise Control of DNA Orientation for Improved Functionlity in Protein Binding Microarrays. In 16th International Conference on Optical MEMS and Nanophotonics; IEEE, 2011; pp 91-92.

Cheng Zhu; Williams, T. E.; Chesla, S. E. Surface Roughness and Molecular Orientation Strongly Influence the Forward but Not the Reverse Rates of Cell-Bound Receptor-Ligand Binding. In Proceedings of the First Joint BMES/EMBS Conference. 1999 IEEE Engineering in Medicine and Biology 21st Annual Conference and the 1999 Annual Fall Meeting of the Biomedical Engineering Society (Cat. No.99 $\mathrm{CH}_{37015)}$; IEEE, 1999; Vol. 1, p 49.

Tremel, K.; Ludwigs, S. Morphology of $\mathrm{P}_{3} \mathrm{HT}$ in Thin Films in Relation to Optical and Electrical Properties. In Romanian Reports of Physics; 2014; Vol. 54, pp 39-82.

Pienpinijtham, P.; Proniewicz, E.; Kim, Y.; Ozaki, Y.; Lombardi, J. R.; Proniewicz, L. M. Molecular Orientation of Neurotensin and Its Single-Site Mutants on a Colloidal Silver Surface: SERS Studies. J. Phys. Chem. C 2012, 116, 16561-16572. 
(20) Knaapila, M.; Winokur, M. J. Structure and Morphology of Polyfluorenes in Solutions and the Solid State. In Polyfluorenes; Springer Berlin Heidelberg: Berlin, Heidelberg, 2009; Vol. 54, pp 227-272.

(21) Campoy-Quiles, M.; Etchegoin, P. G.; Bradley, D. D. C. On the Optical Anisotropy of Conjugated Polymer Thin Films. Phys. Rev. B - Condens. Matter Mater. Phys. 2005, 72, 1-16.

(22) Rivnay, J.; Mannsfeld, S. C. B.; Miller, C. E.; Salleo, A.; Toney, M. F. Quantitative Determination of Organic Semiconductor Microstructure from the Molecular to Device Scale. Chem. Rev. 2012, 112, 5488-5519.

(23) McNeill, C. R. Imaging the Domain Structure of Organic Semiconductor Films. J. Polym. Sci. Part B Polym. Phys. 2011, 49, 909-919.

(24) Wang, X.; Broch, K.; Scholz, R.; Schreiber, F.; Meixner, A. J.; Zhang, D. Topography-Correlated Confocal Raman Microscopy with Cylindrical Vector Beams for Probing Nanoscale Structural Order. J. Phys. Chem. Lett. 2014, 5, 1048-1054.

(25) Chizhik, A. M.; Berger, R.; Chizhik, A. I.; Lyubimtsev, A.; Viani, L.; Cornil, J.; Bär, S.; Hanack, M.; Hulliger, J.; Meixner, A. J.:Egelhaaf, H-S.; Gierschner, J. Polarized Fluorescence from Single Stopcock Molecules at Channel Entrances of an All-Organic Host-Guest Compound. Chem. Mater. 2011, 23, 1088-109o.

(26) Fang, Y.; Zhang, Z.; Chen, L.; Sun, M. Near Field Plasmonic Gradient Effects on High Vacuum Tip-Enhanced Raman Spectroscopy. Phys. Chem. Chem. Phys. 2015, 17, 783-794.

(27) Dietze, D. R.; Mathies, R. A. Molecular Orientation and Optical Properties of 3,3'-Diethylthiatricarbocyanine Iodide Adsorbed to Gold Surfaces: Consequences for SurfaceEnhanced Resonance Raman Spectroscopy. J. Phys. Chem. C 2015, 119, 9980-9987.

(28) Muniz-Miranda, M.; Puggelli, M.; Ricceri, R.; Gabrielli, G. SERS and Molecular Orientation in Langmuir-Blodgett Films Deposited onto Smooth Copper Surfaces. Langmuir 1996, 12, 4417-4420.

(29) Chulhai, D. V.; Jensen, L. Determining Molecular Orientation With Surface-Enhanced Raman Scattering Using Inhomogenous Electric Fields. J. Phys. Chem. C 2013, 130911133503005 .

(30) Fletcher, M. C.; Alexson, D. M.; Prokes, S. M.; Glembocki, O. J.; Vivoni, A.; Hosten, C. M. Determination of Molecular Orientation and Order of N-(6Mercaptoacetylhexyl)Quinolinium
Tricyanoquinodimethanide Adsorbed on Ag Nanoparticles. J. Colloid Interface Sci. 2015, 450, 54-61.

(31) Fletcher, M. C.; Alexson, D. M.; Prokes, S. M.; Glembocki, O. J.; Vivoni, A.; Hosten, C. M. A Surface-Enhanced Raman Study of N-Methylquinolinium Tricyanoquinodimethanide Adsorbed on Ag Nanospheres: Determination of Molecular Orientation and Order. Spectrochim. Acta - Part A Mol. Biomol. Spectrosc. 2015, 147, 286-292.

(32) Marshall, A. R. L.; Stokes, J.; Viscomi, F. N.; Proctor, J. E.; Gierschner, J.; Bouillard, J.-S. G.; Adawi, A. M. Determining Molecular Orientation via Single Molecule SERS in a Plasmonic Nano-Gap. Nanoscale 2017, 9, 17415-17421.

(33) Clément, J. E.; Leray, A.; Bouhelier, A.; Finot, E. Spectral Pointillism of Enhanced Raman Scattering for Accessing Structural and Conformational Information on Single Protein. Phys. Chem. Chem. Phys. 2017, 19, 458-466.

(34) Han, X. X.; Zhao, B.; Ozaki, Y. Surface-Enhanced Raman Scattering for Protein Detection. Anal. Bioanal. Chem. 2009, 394, 1719-1727.

(35) Walter, M. J.; Lupton, J. M.; Becker, K.; Feldmann, J.; Gaefke, G.; Ho, S. Simultaneous Raman and Fluorescence Spectroscopy of Single Conjugated Polymer Chains. Phys. Rev. Lett. 2007, 98, 137401.

(36) Wang, Z.; Rothberg, L. J. Structure and Dynamics of Single Conjugated Polymer Chromophores. ACS Nano 2007, 1, 299306.

(37) Frisch, M. J. Gaussian 09. 2009, p Gaussian, Inc.

(38) Milián-Medina, B.; Gierschner, J. $\pi$-Conjugation. Wiley Interdiscip. Rev. Comput. Mol. Sci. 2012, 2, 513-524.

(39) Itoh, T.; Yamamoto, Y. S. Recent Topics on Single-Molecule Fluctuation Analysis Using Blinking in Surface-Enhanced Resonance Raman Scattering: Clarification by Electromagnetic Mechanism. Analyst 2016, 5000-5009.

(40) Wang, Z.; Rothberg, L. J. Origins of Blinking in SingleMolecule Raman Spectroscopy. J. Phys. Chem. 2005, 8, 33873391.

(41) Kim, N. J. Physical Origins of Chemical Enhancement of Surface-Enhanced Raman Spectroscopy on a Gold Nanoparticle-Coated Polymer. J. Phys. Chem. C 2010, 114, 13979-13984.

(42) Wan, L.-J.; Terashima, M.; Noda, H.; Osawa, M. Molecular Orientation and Ordered Structure of Benzenethiol Adsorbed on Gold(111). J. Phys. Chem. B 200o, 104, 3563-3569. 
Table of Contents artwork

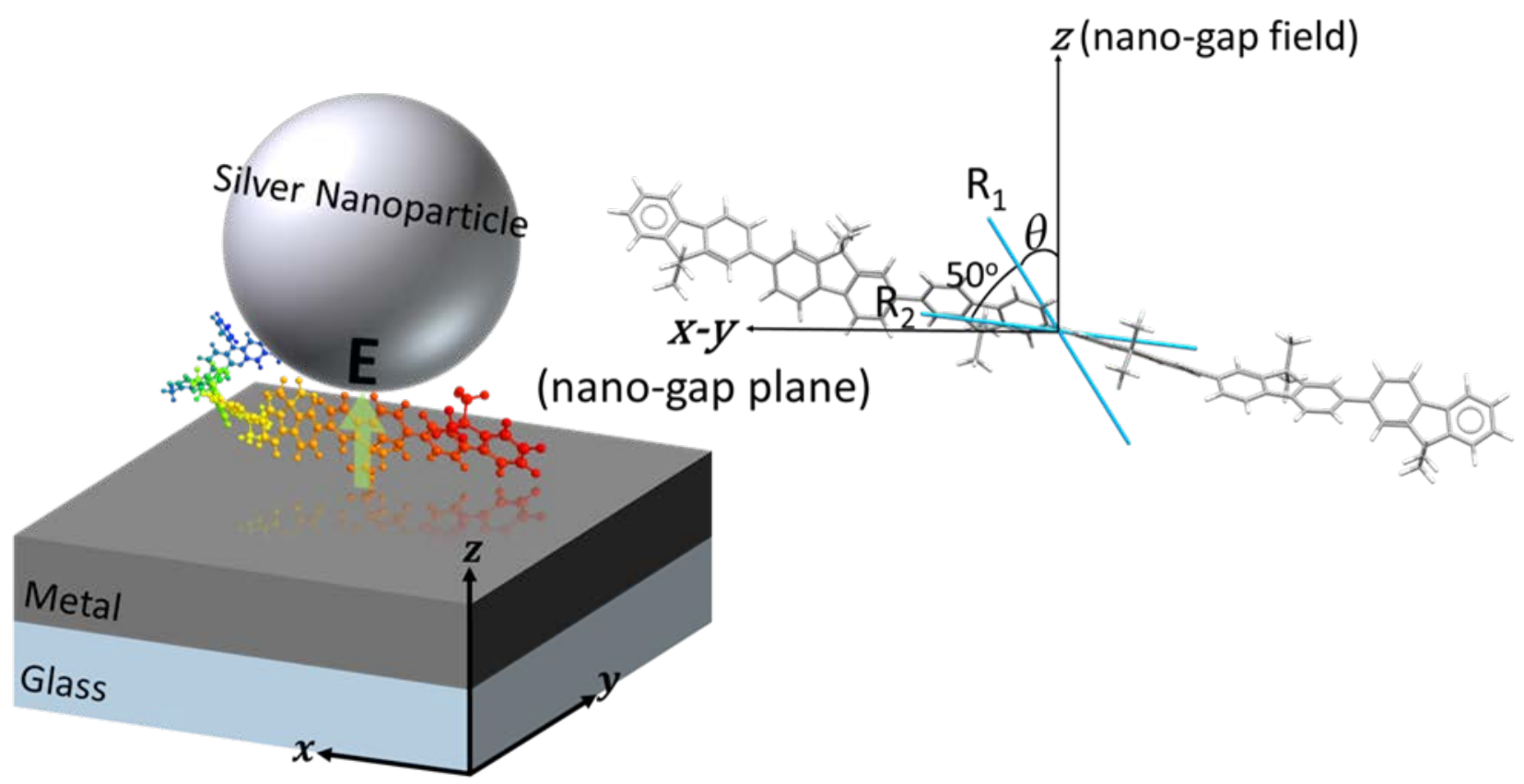

